\title{
Novel azo-peresters radical initiators used for the synthesis of acrylic pressure-sensitive adhesives
}

\author{
Z. Czech 1 , A.Butwin 1 , E. Herko', B. Hefczyc ${ }^{2, *}$, J. Zawadiak $^{2}$ \\ ${ }^{1}$ Department of Chemical Organic Technology, Szczecin University of Technology, Pulaskiego 10, 70-322 Szczecin, \\ Poland \\ 2Department of Organic Chemical Technology and Petrochemistry, Silesian University of Technology, Krzywoustego 4, \\ 44-100 Gliwice, Poland
}

Received 28 January 2008; accepted in revised form 29 February 2008

\begin{abstract}
Acrylic pressure-sensitive adhesives (PSA) based on two monomers: 2-ethylhexyl acrylate and acrylic acid were synthesized in organic solvent ethyl acetate using AIBN (2, 2'-azo-diisobutyronitrile) and new synthesized azo-peresters as radical initiators. After polymerization the viscosity, molecular weight and polydispersity of synthesized acrylic PSA were evaluated. The novel synthesized radical azo-perester initiators were synthesized, isolated and compared with industrial predominant usable azo-initiator AIBN.
\end{abstract}

Keywords: polymer synthesis, radical azo-perester initiators, viscosity, molecular weight, polydispersity

\section{Introduction}

The difference between pressure-sensitive adhesives and other adhesives, such as contact adhesives, is in the permanent surface stickiness of the pressure-sensitive adhesives before, or after, the application. In the giant field of adhesives the pressure-sensitive adhesives make up but a low percentage and the solvent-borne pressure-sensitive acrylic adhesives with their $20000 \ldots 30000$ tons per annum in Europe are almost a quantity negligible within this group. Pressure-sensitive adhesive acrylics can be applied in forms as a solvent-borne, as a water-borne (dispersions) and as a solvent-free system.

Solvent-based acrylic pressure-sensitive adhesives represent more than $45 \%$ of the total PSA produced. Pressure-sensitive adhesive acrylic solutions are nowadays predominantly manufactured by polymerization from a wide selection of monomers containing acrylic groups, often with low lev- els of monomers having pendant functional groups in a refluxing organic solvent in the present of a radical initiator, such as organic peroxides or azo compounds. The acrylic monomers are commonly polymerized utilizing free radical polymerization processes involving the unsaturation contained in the acrylic monomer [1].

Azo groups containing initiators such as azo-peroxy compounds can be divided into: azo-dialkyl peroxides, azo-diacyl peroxides, azo-peresters and azo-hydroperoxides. Fist three groups of azo-peroxy compounds can play a role of bifunctional initiators in generation of block copolymers. They can also play a role of traditional initiator in radical polymerization of just one type of monomers.

Azo-diacyl peroxides and azo-peresters were tested as initiators in styrene [2-3] and acrylamide [4] polymerization processes and in preparation of block copolymers from vinyl [5-7] and acrylic [8] monomers. 


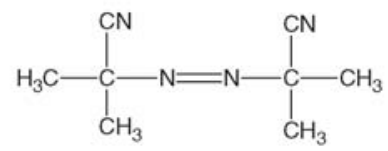

AIBN

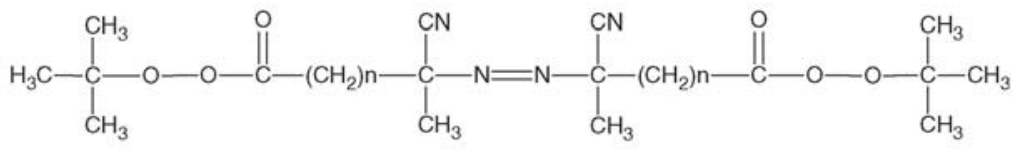

$\mathrm{n}=2:$ AIBN-PEN

3: AIBN-HEX

4: AIBN-HEP

Figure 1. Chemical formula of AIBN and of azo-peresters

In 80's one of azo-peroxy compounds [6] was produced by Ludicol Division of Pennwalt Corporation. This compound was made from commercially available 4,4'-azobis-(4-cyanopentanoic) acid and tert-butyl hydroperoxide. Actually they are not commercially available. Due to the similarity of azo and peroxy groups decomposition temperatures those compounds were not suitable to obtain welldefined block copolymers when both stages of polymerization were initiated thermally. Azo-peroxy compounds in comparioson to traditional free radical initiators have some other advantages, such as higher initiation rate [9].

Compounds described in this article belong to azoperesters group. Those compounds have three labile groups: azo and two peroxy separated wih carbon chain with different number of methylene groups (Figure 1). Figure 1 shows commercial radical initiator AIBN too.

\section{Experimental}

\subsection{Synthesis of azo-peresters radical initiators}

The simpler method to obtain azo-peresters is a method described in this article consisting a single step reaction of carboxylic acid with azo function with tert-butyl hydroperoxide in presence of $1,1^{\prime}$ carbonylimizadazole (Figure 2).

\subsection{Preparation of azo-peroxyesters (general procedure)}

To the 3-RB flask equipment with mechanic stirrer, nitrogen supply and dropping funnel, was intro-

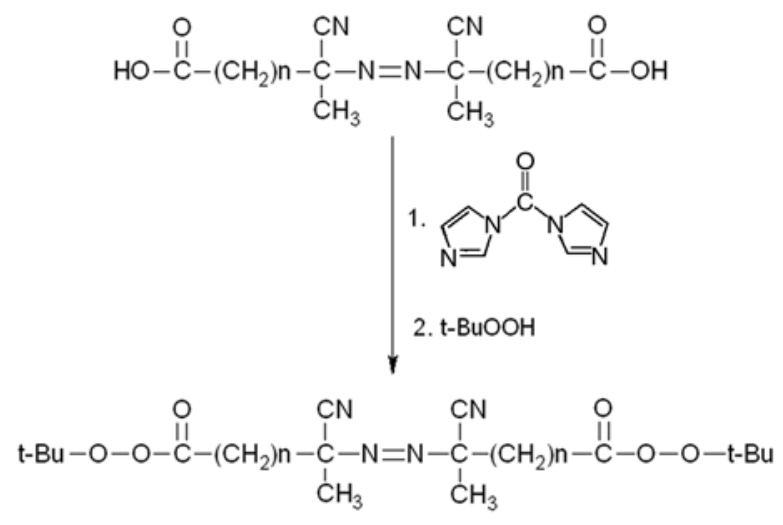

Figure 2. Synthesis of azo-peresters radical initiators

duced a solution of carboxylic acid with azo function $(10 \mathrm{mmol})$ in dry THF. To this mixture was slowly added solution of 1,1'-carbonyldiimidazole (21 mmol) in dry THF. A suspension was extensively stirred for about 30 minutes. After this time the mixture was cooled down to less than $5^{\circ} \mathrm{C}$, and $80 \%$ tert-butyl hydroperoxide $(35 \mathrm{mmol})$ was added. The progress of the reaction was studied by means of TLC (thin layer chromatography) method (mobile phase $\mathrm{CH}_{2} \mathrm{Cl}_{2}: \mathrm{CH}_{3} \mathrm{COCH}_{3}$ 9:1, a solution of sodium iodide in acetic acid was used for visualization of the separated substances). The reaction was continued for $5 \mathrm{~h}$. After this time to reaction mixture was added $\mathrm{Et}_{2} \mathrm{O}$, and stirring was continued for 45 minutes. The mixture was then washed twice: with $5 \% \mathrm{NaOH}$ and water. The organic layer was dried with $\mathrm{MgSO}_{4}$, and then the solvent was evaporated on vacuum rotary of ambient temperature. All azo-peresters were crystallized from $\mathrm{CH}_{2} \mathrm{Cl}_{2}$. 


\subsection{Materials for synthesis of azo-peroxyesters}

Tert-butyl hydroperoxide (Merck) was extracted with hexane. THF was distilled over metallic $\mathrm{Na}$ and used immediately. 4,4'-azobis-(4-cyanopentanoic) acid (Fluka), 1,1'-carbonyldiimidazole (Alfa Aestar), 5,5'-azobis-(5-cyanohexanoic) acid and 6,6'-azobis-(6-cyanoheptanoic) acid were synthesized as described by Silesian University of Technology.

\subsection{Analysis of synthesis of azo-peroxyesters}

${ }^{1} \mathrm{H}$ NMR spectra were recorded in $\mathrm{CDCl}_{3}$ on a Varian Unity Inova-300 spectrometer using TMS as an internal standard and compared, with the reference standard. IR spectra were recorded on a Zeiss Specord M 80 spectrometer.

\subsection{Synthesis of acrylic PSA using synthesized azo-peresters}

The following experiments were conducted to study the influence of kind and concentration of novel azo-peroxyesters as radical initiators on the main properties of synthesized solvent-borne acrylic pressure-sensitive adhesives (PSA) as viscosity, molecular weight and polydispersity [10].

\subsubsection{Materials}

The acrylate monomers, i.e. 2-ethylhexyl acrylate, acrylic acid and solvent ethyl acetate were avail- able from BASF (Germany). AIBN was supplied by Evonik Degussa (Germany) and other investigated radical azo-perester initiators (Figure 3) were synthesized at the Silesian University of Technology.

\subsubsection{Polymerization process}

All starting materials such as acrylate monomers, solvent and AIBN were obtained technical grade and were used without further purification unless otherwise noted. The investigated synthesized azoperoxyesters were likewise technical commercial grade.

The basic solvent-borne pressure-sensitive adhesive was synthesized from between 90 and $99 \mathrm{wt} \%$ of 2-ethylhexyl acrylate and between 1 and $10 \mathrm{wt} \%$ of acrylic acid in ethyl acetate at the boiling point temperature about $77^{\circ} \mathrm{C}$. AIBN and other azo-peroxyesters mentioned in Figure 3 were used as thermal initiators to start radical polymerization in the amount of $0.1 \mathrm{wt} \%$ according to monomers amount. The polymerization process was carried out for $8 \mathrm{~h}$ to reach $50 \mathrm{wt} \%$ of acrylic polymer content [11].

\subsection{Method of testing}

The viscosity of the evaluated solvent-borne acrylic pressure-sensitive adhesives containing the novel azo-peroxyesters initiators was determined with a Rheomat RM 189 from Rheometric Scientific, with spindle No 3 at $23^{\circ} \mathrm{C}$

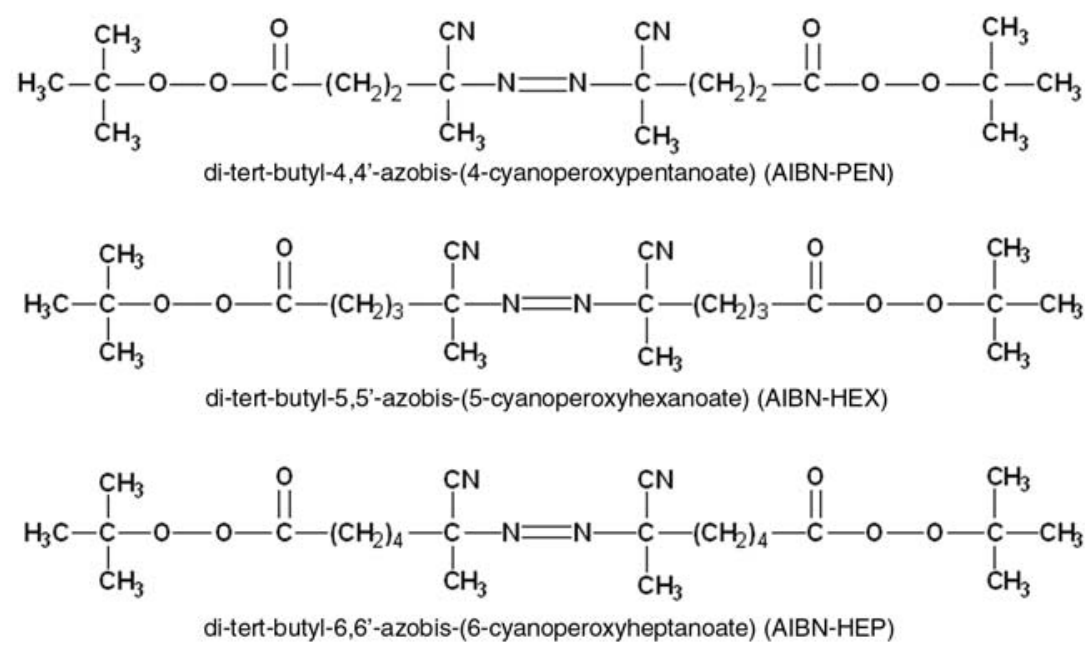

Figure 3. Investigated novel azo-peroxyesters 
The molecular weight studies were performed in tetrahydrofurane with a liquid chromatograph LaChrom system: RI Detector L-7490 and LaChrom UV Detector L-7400 from Merck-Hitachi, equipped with a PLgel $10^{6} \AA$ column from Hewlett-Packard.

\section{Results and discussion}

\subsection{Synthesized azo-peroxyesters}

Azo-peresters were obtained in single step reaction of carboxylic acid possing azo function with tertbutyl hydroperoxide in presence of $1,1^{\prime}$-carbonyldiimidazole. Described method of synthesis is simpler than the double step method, due to the lack of individual preparation and separation of acylating agent (acid chloride). This method was used earlier in synthesis of esters and peresters [12]. Higher yields of desired compounds were achieved by using higher excess of hydroperoxide, than described in the literature [12].

In this method acylating agent is generated in situ by reaction of $1,1^{\prime}$-carbonyldiimidazole with carboxylic acid with azo function. This acylating agent is coupling immediately with hydroperoxide. Yields of obtained azo-perester are higher than obtained in the double step reaction, and purities are comparable (Table 1). All compounds were characterized by comparing their spectra with reference standards.

However one big disadvantage of described method is the sensitivity of $1,1^{\prime}$-carbonyldiimiazole and of the generated acylating agent to traces of water. Presence of water causes the decomposition of those compounds. Azo acid chlorides used in the two step method are also sensitive to water, but hydrolysis of this compound proceeds much slower. Azo acid chlorides are less sensitive to the presence of water than $1,1^{\prime}$-carbonyldiimiazole and acylating agent.

Table 1. Yield of the obtained compounds

\begin{tabular}{l|c|}
\hline \multicolumn{1}{|c|}{ Compound } & $\begin{array}{c}\text { Yield* } \\
{[\%]}\end{array}$ \\
\hline di-tert-butyl-4,4'-azobis-(4-cyanoperoxypentanoate) & 83 \\
\hline di-tert-butyl-5,5'-azobis-(5-cyanoperoxyhexanoate) & 78 \\
\hline di-tert-butyl-6,6'-azobis-(6-cyanoperoxyheptanoate) & 75 \\
\hline *the yield was calculated for pure compounds, determined \\
according to the gravimetric method
\end{tabular}

\subsection{Influence of the kind and concentration of the novel azo-peroxyesters and acrylic acid content on viscosity, molecular weight and polydisperisty of synthesized acrylic PSA}

The effect of acrylic acid concentration (from 1.0 to $10 \mathrm{wt} \%)$ on viscosity, molecular weight and polydispersity of synthesized solvent-borne acrylic pressure-sensitive adhesives is listed in Table 2 and presented in Figures 4-7.

Good examples of the correlations between viscosity, molecular weight and polydispersity of synthesized acrylic PSAs are shown in Table 2. The use of AIBN-HEP with the longest structure of organic radical gives excellent high viscosity and high molecular weight at lowest polydispersity. High viscosity and high molecular weight of acrylic PSAs mean the high content of the long acrylic polymer chains and a relatively small amount of short acrylic chains. This observed effect means the reduction of polydispersity, characterized by low polydispersity values. Acrylic PSAs with low polydispersity are characterized by high $\bar{M}_{w}$ and $\bar{M}_{n}$ values.

The viscosity of synthesised acrylic pressure-sensitive adhesives is very sensitive to the variations of the azo-peroxyester radical initiator kind and concentration of acrylic acid. In general, the viscosity of the acrylic PSA increases clearly with increasing the acrylic acid concentration. The highest viscosity of synthesized acrylic PSA was observed for azo-peroxyester AIBN-HEP with the longest structure of organic radical. The increasing of radical sluggishness influences positively the high molecular weight of synthesized acrylic PSA.

Figure 5 shows the influence of the acrylic acid concentration and kind of azo-peroxyesters and AIBN on weight average molecular weight $M_{w}$ of the synthesized solvent-borne acrylic pressure-sensitive adhesives. As expected after evaluation of viscosity, the increase of acrylic acid concentration corresponds to the molecular weight $M_{w}$ increase of synthesized acrylic PSA. By using of di-tert-butyl6,6'-azobis-(6-cyanoperoxyheptanoate) (AIBNHEP) the highest molecular weight $M_{w}$ performance were observed.

The influence of the acrylic acid concentration and type of the investigated azo-peroxyesters used for polymerization on the number average molecular 
Table 2. Viscosity, molecular weight and polydispersity of synthesized acrylic PSAs produced with AIBN and tested azoperoxyester initiators

\begin{tabular}{|c|c|c|c|c|}
\hline $\begin{array}{c}\text { Concentration } \\
\text { of acrylic acid [wt\%] }\end{array}$ & Viscosity[Pa·s] & $\bar{M}_{w}$ [dalton] & $\bar{M}_{n}$ [dalton] & $P_{d}=\frac{\bar{M}_{w}}{\bar{M}_{n}}$ \\
\hline \multicolumn{5}{|c|}{$0.1 \mathrm{wt} \%$ of $2,2^{\prime}$-azo-bis-diisobutyronitrile (AIBN) } \\
\hline 1.0 & 1.5 & 167000 & 41300 & 4.04 \\
\hline 3.0 & 2.1 & 198000 & 49700 & 3.98 \\
\hline 5.0 & 4.4 & 234000 & 64100 & 3.65 \\
\hline 7.0 & 8.5 & 416000 & 125000 & 3.33 \\
\hline 10.0 & 14.1 & 665000 & 213000 & 3.12 \\
\hline \multicolumn{5}{|c|}{$0.1 \mathrm{wt} \%$ of di-tert-butyl-4,4'-azobis-(4-cyanoperoxypentanoate) (AIBN-PEN) } \\
\hline 1.0 & 4.2 & 210000 & 57500 & 3.65 \\
\hline 3.0 & 4.8 & 255000 & 73500 & 3.47 \\
\hline 5.0 & 6.3 & 321000 & 98800 & 3.25 \\
\hline 7.0 & 9.5 & 509000 & 163000 & 3.12 \\
\hline 10.0 & 16.8 & 765000 & 254000 & 3.01 \\
\hline \multicolumn{5}{|c|}{$0.1 \mathrm{wt} \%$ of di-tert-butyl-5,5'-azobis-(5-cyanoperoxyhexanoate) (AIBN-HEX) } \\
\hline 1.0 & 5.1 & 276000 & 77700 & 3.55 \\
\hline 3.0 & 6.4 & 325000 & 95600 & 3.40 \\
\hline 5.0 & 8.3 & 403000 & 127000 & 3.17 \\
\hline 7.0 & 12.4 & 548000 & 181000 & 3.03 \\
\hline 10.0 & 18.6 & 834000 & 290000 & 2.88 \\
\hline \multicolumn{5}{|c|}{$0.1 \mathrm{wt} \%$ of di-tert-butyl-6,6'-azobis-(6-cyanoperoxyheptanoate) (AIBN-HEP) } \\
\hline 1.0 & 5.8 & 288000 & 86000 & 3.35 \\
\hline 3.0 & 7.3 & 365000 & 113000 & 3.23 \\
\hline 5.0 & 9.9 & 523000 & 170000 & 3.08 \\
\hline 7.0 & 14.4 & 687000 & 239000 & 2.87 \\
\hline 10.0 & 20.7 & 934000 & 347000 & 2.69 \\
\hline
\end{tabular}

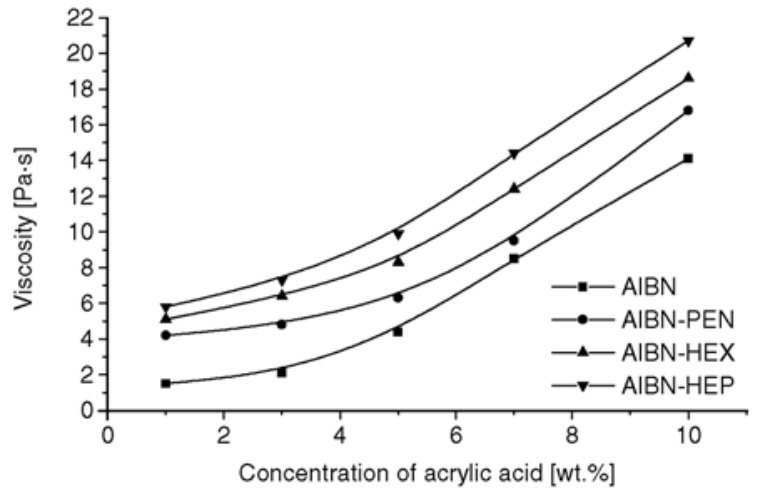

Figure 4. Effect of acrylic acid concentration on viscosity of acrylic PSA synthesized by using of evaluated azo initiators

weight $M_{n}$ of the synthesized solvent-borne acrylic pressure-sensitive adhesives is described in Figure 6. As can be seen from the $M_{n}$ results recorded in Figure 6, the efficiency of the evaluated radical azo-peroxyester initiators of acrylic PSA molecular weight clearly corresponds to their chemical architecture, expressed as length of azo-peroxyester molecule. The longer the azo-peroxyester molecule, the greater the acrylic PSA molecular weight (AIBN-HEP). With the increase of acrylic acid

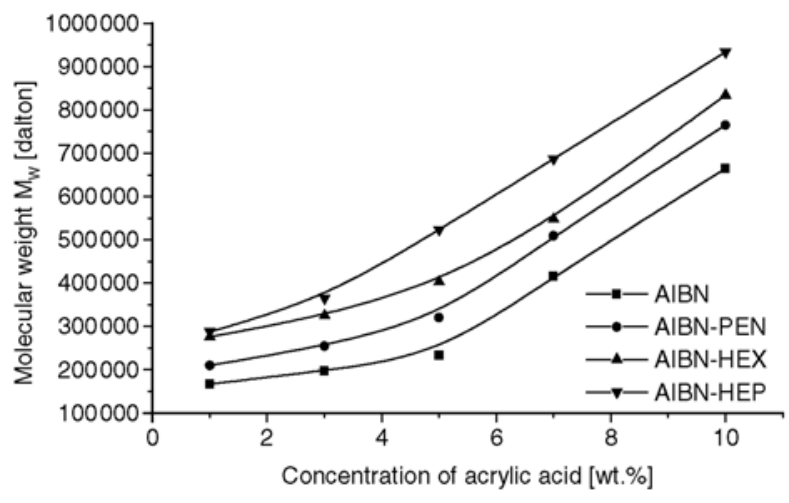

Figure 5. Effect of acrylic acid concentration on molecular weight $M_{w}$ of acrylic PSA synthesized by using of evaluated azo initiators

amount the molecular weight $M_{n}$ of synthesized acrylic PSA also rises.

Higher molecular weight adhesives allow the manufacture of cohesive layers and lower molecular weight adhesives the manufacturing of adhesive and tacky layers. Acrylic PSA polydispersity as molecular weight distribution includes information about acrylic soft and hard performance.

Figure 7 shows polydispersity of the synthesized acrylic PSA as a function of different concentration 


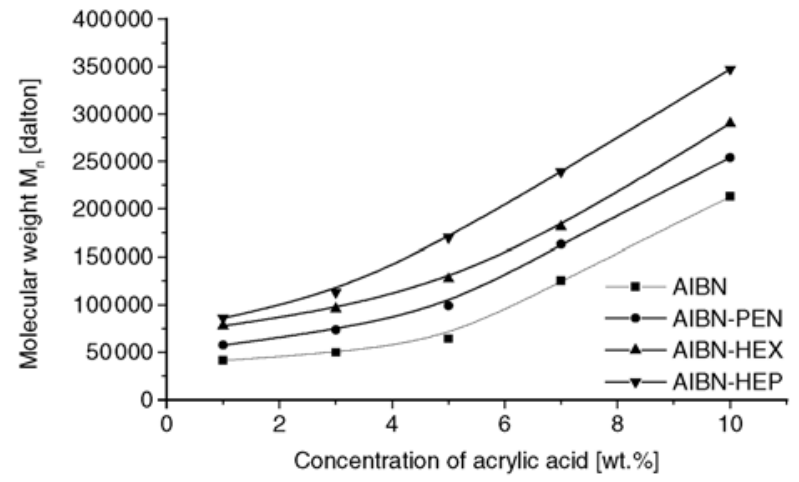

Figure 6. Effect of acrylic acid concentration on molecular weight $M_{n}$ of acrylic PSA synthesized by using of evaluated azo initiators

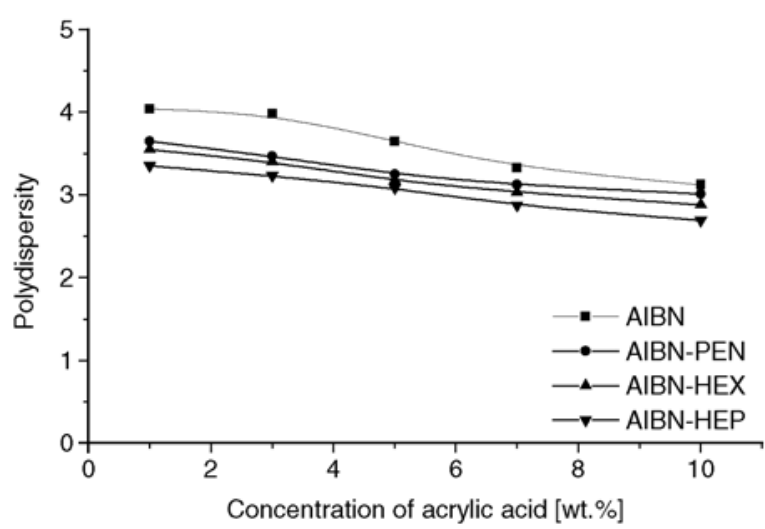

Figure 7. Effect of acrylic acid concentration on the polydispersity of acrylic PSA synthesized by using of evaluated azo initiators

of acrylic acid and kind of novel azo-peroxyesters initiators and AIBN. The following polydispersity ranking for various concentrations of acrylic acid and type of investigated azo initiators was obtained: 2,2'-azo-bis-diisobutyronitrile (AIBN), di-tert-butyl-4,4'-azobis-(4-cyanoperoxypetanoate) (AIBN-PEN), di-tert-butyl-5,5'-azobis-(5-cyanoperoxyhexanoate) (AIBN-HEX) and di-tert-butyl6,6'-azobis-(6-cyanoperoxy-heptanoate) (AIBNHEP). For all synthesized acrylic PSA containing different contents of acrylic acid the using of AIBN allows synthesis of solvent-borne acrylic PSA with high tack and adhesion properties and the using of novel azo-peroxyesters initiators allows manufacturing of acrylic PSA characterized by high cohesion level.

\section{Conclusions}

The evaluation of novel synthesized azo-peroxyesters initiators has recently attracted considerable attention, due to their practical and potential application in various industrial branches to comprehensive permanent self-adhesive technical and medical articles with the wide range of very interesting original performance. Solvent-borne acrylic pressuresensitive adhesives with high performance can be formed by the radical polymerization of typical acrylate monomers such as 2-ethylhexyl acrylate and acrylic acid using instead of AIBN the novel azo-peroxyester initiators. Especially the di-tertbutyl-6,6'-azobis-(6-cyanoperoxy-heptanoate)

(AIBN-HEP) guarantees satisfactorily performance with respect to the important properties of solventborne acrylic PSA containing various amounts of acrylic acid between 3 and $7 \mathrm{wt} \%$.

\section{References}

[1] Czech Z.: Stable vinyl-oxy-carbonyl-oxy and -amino compounds with chromophore group. German patent, DE19501025, Germany (1996).

[2] Simionescu C., Sik K. G., Comaniţa E., Dumitriu S.: Bifunctional initiators, VI. Polymerization of styrene with 4,4-azo-bis-(4-cyanovaleryl)-bis-(m-chlorobenzoyl)-diperoxide as initiator. Die Angewandte Makromolekulare Chemie, 126, 73-80 (1984).

[3] Simionescu C. I., Popa A. A., Comaniţla E., Comaniţla B.: Polymerization of styrene initiated with cumyl 4-tbutylazo-4-cyanoperoxypentanoate. Polymer-Plastics Technology and Engineering, 31, 451-461 (1992).

[4] Sik K.G., Dumitriu S., Comaniţa E., Simionescu C.: Bifunctional initiators: 7. Acrylamide polymerization with 4,4'-azo-bis-(4-Cyanovaleryl)-bis-(m-Chlorobenzoyl) diperoxide as initiator. Polymer Bulletin, 12, 419-425 (1984).

[5] Vogla C., Hazer B., Torul O.: Kinetic study of vinyl polymerization with a new oligo azo peroxidic initiator. European Polymer Journal, 33, 907-912 (1997).

[6] Piirma I., Chou L-P.: Block copolymers obtained by free-radical mechanism, I. Methyl methacrylate and styrene. Journal of Applied Polymer Science, 24, 2051-2070 (1979).

[7] Hazer B., Ayas A., Besirli N., Saltek N., Baysal B. M.: Preparation of ABCBA-type block copolymers by use of macro-initiators containing peroxy and azo groups. Die Makromolekulare Chemie, 190, 1987-1996 (1989).

[8] Gunesin B. Z., Piirma I.: Block copolymers obtained by free radical mechanism, II. Butyl acrylate and methyl methacrylate. Journal of Applied Polymer Science, 26, 3103-3115 (1981).

[9] Simionescu Cr., Comaniţa E., Pãstrãvanu M., Dumitriu S.: Progress in the field of bi- and poly-functional free-radical polymerization initiators. Progress in Polymer Science, 12, 1-109 (1986). 
[10] Czech Z.: Synthesis and cross-linking of acrylic PSA systems. Journal of Adhesion Science and Technology, 7, 625-635 (2007).

[11] Czech Z., Wesolowska M.: Development of solventfree acrylic pressure-sensitive adhesives. European Polymer Journal, 43, 3604-3612 (2007).
[12] Staab H.A., Rohr W., Graf F.: Darstellung von Diacylperoxyden und Persäureestern nach der Imidazolidmethode. Chemische Berichte, 98, 1122-1127 (1965). 